to appear in the Proceedings of the Combustion Institute 31 (2006)

\title{
Computational Model of Forward and Opposed Smoldering Combustion in Microgravity
}

\author{
Guillermo Rein*, A. Carlos Fernandez-Pello \\ University of California Berkeley, CA, USA \\ David L. Urban \\ NASA Glenn Research Center, OH, USA
}

\begin{abstract}
A novel computational model of smoldering combustion capable of predicting both forward and opposed propagation is developed. This is accomplished by considering the one-dimensional, transient, governing equations for smoldering combustion in a porous fuel accounting for improved chemical kinetics. The heterogeneous chemistry is modeled with a 5-step mechanism for polyurethane foam. The kinetic parameters for this mechanism were obtained from thermogravimetric data in the literature and reported by the authors elsewhere. The results from previously conducted microgravity experiments with flexible polyurethane foam are used for calibration and testing of the numerical results. Both forward and opposed smoldering configurations are examined. By considering the 5-step mechanism, the numerical model is able to predict qualitatively and quantitatively the smoldering behavior, reproducing the most important features of the process. Specifically, the model predicts the transient temperature profiles, the overall structure of the reaction-front, the onset of smoldering ignition, and the propagation rate. The fact that it is possible to predict the experimental observations in both opposed and forward propagation with a single model is a significant improvement in the development of numerical models of smoldering combustion. This is particularly relevant in multidimensional simulations where distinction between forward and opposed modes is no longer applicable.
\end{abstract}

Keywords: Smoulder; Polyurethane; Fire; Kinetics; Porous Media

\footnotetext{
* Current affiliation: School of Engineering and Electronics, University of Edinburgh, EH9 3JL, UK.
} 


\begin{abstract}
A novel computational model of smoldering combustion capable of predicting both forward and opposed propagation is developed. This is accomplished by considering the one-dimensional, transient, governing equations for smoldering combustion in a porous fuel accounting for improved chemical kinetics. The heterogeneous chemistry is modeled with a 5-step mechanism for polyurethane foam. The kinetic parameters for this mechanism were obtained from thermogravimetric data in the literature and reported by the authors elsewhere. The results from previously conducted microgravity experiments with flexible polyurethane foam are used for calibration and testing of the numerical results. Both forward and opposed smoldering configurations are examined. By considering the 5-step mechanism, the numerical model is able to predict qualitatively and quantitatively the smoldering behavior, reproducing the most important features of the process. Specifically, the model predicts the transient temperature profiles, the overall structure of the reaction-front, the onset of smoldering ignition, and the propagation rate. The fact that it is possible to predict the experimental observations in both opposed and forward propagation with a single model is a significant improvement in the development of numerical models of smoldering combustion. This is particularly relevant in multidimensional simulations where distinction between forward and opposed modes is no longer applicable.
\end{abstract}

Keywords: Smoulder; Polyurethane; Fire; Kinetics; Porous Media 


\begin{tabular}{ll} 
Nomenclature & \\
$\mathrm{A}_{\mathrm{gs}} / \mathrm{V}$ & Preexponential factor \\
$\mathrm{A}_{\mathrm{L}} / \mathrm{V}$ & Ratio of surface area between gas and solid to volume \\
$\mathrm{c}$ & Ratio of lateral area to volume \\
$\mathrm{d}_{\mathrm{f}}$ & Fibecific heat \\
$\mathrm{d}_{\mathrm{p}}$ & Pore diameter \\
$\mathrm{D}$ & Diameter of fuel sample \\
$\mathrm{E}$ & Activation energy \\
$\Delta \mathrm{h}$ & Enthalpy of reaction \\
$\mathrm{h}^{\prime \prime}$ & Enthalpy per unit volume \\
$\mathrm{h}_{\mathrm{gs}}$ & Heat transfer coefficient between gas and solid \\
$\mathrm{K}$ & Permeability \\
$\mathrm{k}$ & Conductivity \\
$\mathrm{L}$ & Sample length \\
$\mathrm{l}_{\mathrm{m}}$ & Mean penetration distance \\
$\mathrm{m}$ & Fraction of mass of solid species respect to initial total mass \\
\hline $\mathrm{MW}$ & Average molecular weight \\
$\mathrm{n}$ & Reaction order for solid reactant \\
$\mathrm{p}$ & Pressure \\
$\mathrm{T}$ & Temperature \\
$\mathrm{u}$ & Velocity \\
$\mathrm{U}_{\mathrm{e}}$ & Global heat-loss coefficient to exterior \\
$\mathrm{y}$ & Mass fraction of gas species \\
&
\end{tabular}

\section{Greek letters}

$v$

$\rho$

Mass yield/consumption of species per mass of reactant

Density

$\rho_{0} \quad$ Density of the initial fuel sample

$\sigma \quad$ Stephan-Boltzmann constant

$\phi \quad$ Porosity of the media

ஸ) Reaction rate

\section{Subscripts}

0

Initial or ambient conditions

Char solid species / Char oxidation reaction

Foam solid species

Gas

g

Other gas products species

gp

Foam oxidation reaction

o $\beta$

$\beta$-foam oxidation reaction

$\mathrm{p}$

Foam pyrolysis reaction

$\mathrm{p} \beta$

$\beta$-foam pyrolysis reaction

$\mathrm{r}$

Residue solid species

$\beta$

$\beta$-foam solid species 


\section{Introduction}

Smoldering combustion is controlled by strong interactions between heat, mass and momentum transports and heterogeneous chemical reactions in porous media [1]. Numerical models of the ignition and propagation of smoldering combustion provide means of identifying and quantifying the smoldercontrolling mechanisms and are especially useful to understand experimental observations. In addition, they are a cost-effective complement to experimentation, in particular under special circumstances as it is the case in microgravity environments. Space-based smoldering experiments are scarce and unique because of their high cost and consequently it is of great importance to use modeling approaches to extend the limited microgravity data to different configurations, thermal and flow conditions, and fuels. Transient one-dimensional models, while using a simplified representation of the spatial domain, are able to reproduce the interactions between the controlling phenomena with accuracy and to provide useful insights of the process, e.g. [2, 3, 4, 5, 6, 7].

The propagation rate of self-sustained smoldering is typically controlled by oxygen transport and net heat losses [1, 8]. However, heterogeneous chemical kinetics governs the front structure and dictates the global heat-released rate. Proper computation of the reaction rates is particularly essential when modeling the kinetically controlled regimes of ignition, extinction, and the transition to flaming. Unfortunately, it is difficult to establish and quantify kinetic mechanisms of solid decomposition with certainty, especially for materials with complex kinetics like polyurethane (PU). This difficulty is one of the biggest impediments preventing an increased usage of models of smoldering combustion [1,7].

One-dimensional smoldering propagation is classified as opposed or forward (Fig. 1). In forward smoldering combustion, the reaction front propagates in the same direction as the oxidizer flow, and in opposed smoldering the front propagates in the opposite direction. These two configurations are distinguished by the different roles that the transport mechanisms and chemical reactions play [9]. Conventional models of smoldering use different kinetic schemes depending on the propagation mode. 
Forward smoldering combustion is generally described using a 2-step mechanism having pyrolysis and oxidation reactions $[3,4,10,11]$, whereas in opposed smoldering these two paths are lumped together in a global single reaction $[12,13,14,15]$. Since there are no fundamental kinetic differences between opposed and forward smoldering combustion, the same appropriate kinetic scheme should describe adequately both forms. Another difficulty with chemical mechanism for smoldering combustion is that valid kinetic parameters for numerical computation are rarely available. The most widespread kinetic mechanism for numerical models of smoldering combustion, the 3-step mechanism proposed by Ohlemiller [1], has been used for cellulose and PU with considerable success [2, 5, 6, 16]. But to date, no study has attempted to simulate both forward and opposed smoldering combustion with the same kinetic mechanism and same kinetic parameters.

Recently, Rein et al. [17] showed that a 5-step mechanism for PU is able to predict at least phenomenologically the reaction structure in both opposed and forward smoldering combustion. This 5step mechanism is implemented here into a detailed model of forward and opposed smoldering ignition and propagation. The results from previously reported microgravity experiments $[8,18]$ with PU as fuel are used for calibration and testing of the numerical results.

\section{Polyurethane Chemical Kinetics}

The 5-step mechanism for PU [17] consists of: two foam-pyrolysis reactions (Eqs. 1 and 2); two foam oxidation reactions (Eqs. 3 and 4); and one char oxidation reaction (Eq. 5), accounting for four solid species: foam, $\beta$-foam, char and residue, and two gas species; oxygen and products of smoldering.

$$
\begin{aligned}
& \text { Foam } \rightarrow \mathrm{v}_{\beta, p} \text { ß-foam }+\mathrm{v}_{g p, p} \text { Gas } \\
& \text { ß-foam } \rightarrow \mathrm{v}_{c, p \beta} \text { Char }+\mathrm{v}_{g p, p \beta} \text { Gas } \\
& \text { Foam }+\mathrm{v}_{O_{2}, o} O_{2} \rightarrow \mathrm{v}_{c, o} \text { Char }+\mathrm{v}_{g p, o} \text { Gas } \\
& \text { В-foam }+\mathrm{v}_{O_{2}, o \beta} O_{2} \rightarrow \mathrm{v}_{c, o \beta} \text { Char }+\mathrm{v}_{g p, o \beta} \text { Gas }
\end{aligned}
$$


Char $+\mathrm{v}_{O_{2}, c} O_{2} \rightarrow \mathrm{v}_{r, c}$ Residue $+\mathrm{v}_{g p, c}$ Gas

The reaction rates for each one of the paths described above are expressed in the Arrhenius form. For a pyrolysis reaction, it is:

$\dot{\omega}_{\mathrm{i}}=\mathrm{A}_{\mathrm{i}} \mathrm{e}^{-\frac{\mathrm{E}_{\mathrm{i}}}{\mathrm{RT}}} \mathrm{m}_{\mathrm{i}}^{{ }_{\mathrm{n}}}$,

and for an oxidation reaction:

$\dot{\omega}_{\mathrm{i}}=\mathrm{A}_{\mathrm{i}} \mathrm{e}^{-\frac{\mathrm{E}_{\mathrm{i}}}{\mathrm{RT}}} \mathrm{m}_{\mathrm{i}}^{\mathrm{n}_{\mathrm{i}}} \mathrm{y}_{\mathrm{O}_{2}}$

which are expressed as a function of $\mathrm{m}_{\mathrm{i}}$ (fraction of mass of solid species $\mathrm{i}$ respect to the initial total mass of the virgin fuel). The corresponding 20 kinetic parameters $\left(A_{i}, E_{i}, n_{i}\right.$ and $\left.v_{i, j}\right)$ have been obtained elsewhere [17] from thermogravimetric experiments of PU in conjunction with a genetic algorithm.

\section{Model of Smoldering Ignition and Propagation}

The computational domain is shown in Fig. 1, which reproduces the conditions in the microgravity experiments as reported by $[8,18]$. The ignition is applied at the boundary $\mathrm{x}=0$. Air is forced at one boundary at a velocity $\mathrm{u}_{0}$ and flows through the domain. For opposed propagation, air is forced at the boundary $\mathrm{x}=\mathrm{L}$, for forward propagation air is forced at $\mathrm{x}=0$.

The model solves the one-dimensional transient equations for the solid and the gas. These equations are developed combining the models in $[2,5,6,16]$, plus some novel contributions. Only the essentials of the model are presented here and details and further results can be found in [19]. It consists of the conservation of energy of the solid (Eq. 8), solid species (Eqs. 9-12), energy of the gas (Eq. 13), continuity of the gas (Eq. 14) and gas species (Eqs. 15 and 16). Darcy's law is used as the equation for the conservation of momentum, Eq. (17), and computes the gas velocity as a linear function of the pressure gradient in the porous medium. Buoyancy-induced flows are not modeled in Eq. (17) and thus the simulations are in microgravity conditions. 


$$
\begin{aligned}
& \frac{\partial \mathrm{h}_{\mathrm{s}}^{\prime \prime \prime}}{\partial \mathrm{t}}=\frac{\partial}{\partial \mathrm{x}}\left(\mathrm{k}_{\mathrm{eff}, \mathrm{s}} \frac{\partial \mathrm{T}_{\mathrm{s}}}{\partial \mathrm{x}}\right)-\rho_{0} \sum_{\mathrm{i}} \dot{\omega}_{\mathrm{i}} \Delta \mathrm{h}_{\mathrm{i}}+\mathrm{h}_{\mathrm{gs}} \frac{\mathrm{A}_{\mathrm{gs}}}{\mathrm{V}}\left(\mathrm{T}_{\mathrm{g}}-\mathrm{T}_{\mathrm{s}}\right)-\mathrm{U}_{\mathrm{e}} \frac{\mathrm{A}_{\mathrm{L}}}{\mathrm{V}}\left(\mathrm{T}_{\mathrm{s}}-\mathrm{T}_{0}\right) \\
& \frac{\partial \mathrm{m}_{\mathrm{f}}}{\partial \mathrm{t}}=-\dot{\omega}_{\mathrm{p}}-\dot{\omega}_{\mathrm{o}} \\
& \frac{\partial \mathrm{m}_{\beta}}{\partial \mathrm{t}}=\mathrm{v}_{\beta, \mathrm{p}} \dot{\omega}_{\mathrm{p}}-\dot{\omega}_{\mathrm{p} \beta}-\dot{\omega}_{\mathrm{o} \beta} \\
& \frac{\partial \mathrm{m}_{\mathrm{c}}}{\partial \mathrm{t}}=\mathrm{v}_{\mathrm{c}, \mathrm{p} \beta} \dot{\omega}_{\mathrm{p} \beta}+\mathrm{v}_{\mathrm{c}, \mathrm{o}} \dot{\omega}_{\mathrm{o}}+\mathrm{v}_{\mathrm{c}, \mathrm{o} \beta} \dot{\omega}_{\mathrm{o} \beta}-\dot{\omega}_{\mathrm{c}} \\
& \frac{\partial \mathrm{m}_{\mathrm{r}}}{\partial \mathrm{t}}=\mathrm{v}_{\mathrm{r}, \mathrm{c}} \dot{\omega}_{\mathrm{c}} \\
& \frac{\partial}{\partial t}\left(\phi h_{g}^{\prime \prime \prime}\right)=\frac{\partial}{\partial x}\left(\phi k_{g} \frac{\partial T_{g}}{\partial x}\right)-\frac{\partial}{\partial x}\left(\phi \rho_{g} u c_{p g}\left(T_{g}-T_{0}\right)\right)-h_{g s} \frac{A_{g s}}{V}\left(T_{g}-T_{s}\right) \\
& \frac{\partial}{\partial t}\left(\phi \rho_{g}\right)+\frac{\partial}{\partial x}\left(\phi \rho_{g} u\right)=\rho_{0} \sum_{i} \dot{\omega}_{i}\left(v_{g, i}-v_{O_{2}, i}\right) \\
& \frac{\partial}{\partial t}\left(\phi \rho_{g} y_{O_{2}}\right)+\frac{\partial}{\partial x}\left(\phi \rho_{g} u y_{O_{2}}\right)=\frac{\partial}{\partial x}\left(\phi \rho_{g} D_{\text {diff }} \frac{\partial y_{O_{2}}}{\partial x}\right)-\rho_{0} \sum_{i} \dot{\omega}_{i} v_{O_{2}, i} \\
& \frac{\partial}{\partial t}\left(\phi \rho_{g} y_{g p}\right)+\frac{\partial}{\partial x}\left(\phi \rho_{g} u y_{g p}\right)=\frac{\partial}{\partial x}\left(\phi \rho_{g} D_{d i f f} \frac{\partial y_{g p}}{\partial x}\right)+\rho_{0} \sum_{i} \dot{\omega}_{i} v_{g p, i} \\
& \mathrm{u}=-\frac{\mathrm{K}}{\mu} \frac{\partial \mathrm{p}}{\partial \mathrm{x}}
\end{aligned}
$$

The pressure is calculated using the ideal gas law:

$\mathrm{p}=\rho_{\mathrm{g}} \frac{\mathrm{RT}_{\mathrm{g}}}{\overline{\mathrm{MW}}}$

The equations for the conservation of energy, Eqs. (8) and (13), are formulated in terms of the enthalpy.

To calculate the temperature of each phase, the following expressions apply:

$$
\mathrm{T}_{\mathrm{s}}=\frac{\mathrm{h}_{\mathrm{s}}^{\prime \prime \prime}}{\mathrm{c}_{\mathrm{s}} \rho_{0} \sum_{\mathrm{i}} \mathrm{m}_{\mathrm{i}}}+\mathrm{T}_{0}
$$




$$
\mathrm{T}_{\mathrm{g}}=\frac{\mathrm{h}_{\mathrm{g}}^{\prime \prime \prime}}{\mathrm{c}_{\mathrm{pg}} \rho_{\mathrm{g}}}+\mathrm{T}_{0}
$$

Where the density of the solid has been expressed in a consistent way with the reaction rates in Eqs. (9-

12) as $\rho_{\mathrm{s}}=\rho_{0} \sum_{\mathrm{i}} \mathrm{m}_{\mathrm{i}}$. The conductivity of the solid $\mathrm{k}_{\mathrm{eff}, \mathrm{s}}$ in Eq. (7) includes the term $\mathrm{k}_{\mathrm{r}}=\frac{3}{16} \mathrm{l}_{\mathrm{m}} \sigma \mathrm{T}_{\mathrm{s}}^{3}$, which is the radiative conductivity in the optically thick limit [20]. According to electron-microscopy photographs of PU foam [19], the mean penetration distance $l_{m}$ is approximately three times the pore diameter.

One setback of one-dimensional simulations of smoldering combustion is that they cannot directly model heat losses to the external environment (in the perpendicular direction), as occurs in actual experiments. However, the effect of these heat losses can be accounted for in an approximate way as a volumetric heatloss coefficient $U_{e}$ in Eq. (8). This coefficient has been analytically calculated elsewhere [8] for the particular experimental configuration used in the smoldering experiments $[8,18]$, and its effective value in microgravity is $0.3 \mathrm{~W} / \mathrm{m}^{2} \mathrm{~K}$. The lateral area to volume ratio in Eq. (8) is given for the cylindrical samples of diameter $\mathrm{D}$ used in the experiments by $A_{L} / V=4 / D$.

The heat transfer between the gas phase and the solid phase is quantified by the multiplication of the heattransfer coefficient $h_{g s}$ and the exchange area to volume ratio $A_{g s} / V$. Scanning electron microscopy (SEM) photographs of the foam [19] provide measurements of the pore and fiber diameters in the virgin foam and char microstructures (values shown in Table 1). A simple estimation of the order of magnitude of the volumetric coefficient can be done using these measurements and a rough model of the geometry of the pores. Assuming that the heat-transfer boundary-layer in the gas is of the order of magnitude of the pore diameter, the heat-transfer coefficient is given by:

$$
\mathrm{h}_{\mathrm{gs}}=\frac{\mathrm{k}_{\mathrm{g}}}{\mathrm{d}_{\mathrm{p}}}
$$

Approximating the pore geometry as hollow cube whose edges are the fibers, the exchange surface to volume ratio is given by: 


$$
\frac{\mathrm{A}_{\mathrm{gs}}}{\mathrm{V}} \approx \frac{12 \mathrm{~d}_{\mathrm{f}}}{\mathrm{d}_{\mathrm{p}}^{2}}
$$

When the geometry values in Table 1 are substituted into this expression, the exchange surface to volume ratio is $41001 / \mathrm{m}$ for the virgin foam. This value is inside the approximate range from 4000 to $50001 / \mathrm{m}$ given by [21] as an experimental estimation for PU. With these expressions and values, the order of magnitude of the corresponding volumetric heat-transfer coefficient $h_{g s} A_{g s} / V$ is $10^{5} \mathrm{~W} / \mathrm{m}^{3} \mathrm{~K}$, which is high enough to imply virtual thermal equilibrium between the gas and the solid during smoldering (as the results show).

Assuming that the solid densities of all the species are similar to that of the virgin foam, then the porosity is given by:

$$
\phi=1-\left(1-\phi_{0}\right) \sum_{\mathrm{i}} \mathrm{m}_{\mathrm{i}}
$$

The rest of the properties of the solid phase $\left(d_{p}, d_{f}\right.$ and $\left.K\right)$ are weight averaged for the four solid species, assuming that the $\beta$-foam has the same properties as the foam, and that the residue has the same properties as the char. The molecular weight of the gas is calculated with the mass fractions of the three gas species $\left(\mathrm{O}_{2}, \mathrm{~N}_{2}\right.$ and gas products of smoldering). Walther et al. [22] reported the composition of the gases collected during PU smoldering experiments. This composition is used to approximate the molecular weight of the products of smoldering with $\mathrm{MW}_{\mathrm{gp}}=39 \mathrm{~g} / \mathrm{mol}$. The mass diffusivity $\mathrm{D}_{\text {diff }}$ is assumed to be that of $\mathrm{O}_{2}$ in air $\left(2.710^{-5} \mathrm{~m}^{2} / \mathrm{s}\right)$. The properties of the gas phase $\left(\mu, c_{\mathrm{pg}}\right.$ and $\left.\mathrm{k}_{\mathrm{g}}\right)$ are approximated to those of air using temperature-dependent correlations. Values for the most important parameters in the model are shown in Table 2.

At $t=0$, the entire fuel bed is considered unreacted and the solid and gas are at ambient temperature (27 $\left.{ }^{\circ} \mathrm{C}\right)$. The heat-flux imposed by the igniter is such that the temperature rise with time at the igniter location is the same as in the experiments (ignition time of $600 \mathrm{~s}$ with a final igniter temperature of $480{ }^{\circ} \mathrm{C}$ for the opposed case [8], and ignition time of $400 \mathrm{~s}$ with a final temperature of $400{ }^{\circ} \mathrm{C}$ for the forward case [18]). 
The thermal boundary condition after the ignition protocol is that heat is lost to the ambient resulting in the same cooling effect as seen in the experiments. As in the experiments, during the ignition, the inlet flow velocity is $0.01 \mathrm{~mm} / \mathrm{s}$. After ignition, the inlet forced-flow velocity $\mathrm{u}_{0}$ is set to the corresponding nominal value and kept constant at the boundary thereafter. This flow condition is implemented as a pressure gradient following Eq. (17). The outlet is at constant ambient pressure.

The spatial partial derivatives in Eqs. (7)-(15) are discretized using explicit finite-differences in a uniform grid. The resulting system of equations consists of nine ODEs per node, one for each of the variables: $\mathrm{h}_{\mathrm{s}}^{\prime \prime \prime}, \mathrm{h}_{\mathrm{g}}^{\prime \prime \prime}, \rho_{\mathrm{g}}, \mathrm{m}_{\mathrm{f}}, \mathrm{m}_{\beta}, \mathrm{m}_{\mathrm{c}}, \mathrm{m}_{\mathrm{r}}, \mathrm{y}_{\mathrm{O}_{2}}$ and $\mathrm{y}_{\mathrm{gp}}$. This system is then solved in time using the stiff integrator VODE [23]. The effect of the spatial-grid size on the results was analyzed by conducting a grid-independence study. The convergence of the results was assessed integrating over the spatial domain the square of the differences between the computed temperature-profile for a given grid and that for the grid of 2000 nodes (in opposed propagation with an airflow of $3 \mathrm{~mm} / \mathrm{s}$ ). The results converge as the grid is increased and the study concludes that a grid of 500 nodes provides satisfactory accuracy (i.e. maximum temperature discrepancy lower than $3{ }^{\circ} \mathrm{C}$ ).

Suitable thermochemistry values for the smoldering combustion of PU are not available in the literature, where only the rough orders of magnitude of some parameters are provided. Moreover, previous chemical studies of flexible PU foam have mainly focused on pyrolysis degradation. As a consequence, there is little experimental information on oxygen consumptions and heats of reaction. For this reason, these unknown parameters are determined here through calibration and comparison of the numerical results with two experiments in microgravity (as shown below). The final values for the thermochemistry parameters are shown in Table 2.

\section{Results and Discussion}

Results for the temperature profiles of the solid and a direct comparison with the experiments are shown in Figs. 2 and 3. For the opposed case with an airflow of $3 \mathrm{~mm} / \mathrm{s}$, the smoldering peak-temperature is 380 
${ }^{\circ} \mathrm{C}$ with a propagation velocity of $0.12 \mathrm{~mm} / \mathrm{s}$. The smoldering peak-temperature for the forward case with an airflow of $5 \mathrm{~mm} / \mathrm{s}$ is $430{ }^{\circ} \mathrm{C}$, and the propagation velocity is around $0.21 \mathrm{~mm} / \mathrm{s}$. In forward propagation, the temperature profiles shows a dip moving ahead of the front that is caused by the endothermic pyrolysis. This dip is not present in the opposed propagation.

While running different cases with the model, it was noted that modifications in the ignition protocol significantly affect the temperature time-histories and profiles. Thus, in order to compare to the experimental thermocouple measurements, it is important to match the thermal and flow conditions.

The forward case is the most difficult to model because two phenomena that are not included into the model took place in the experiments. The first phenomenon is related to the plateaus at about $75{ }^{\circ} \mathrm{C}$ (right of Fig. 3), which are typical of forward propagation and have been attributed to water evaporation [11, 24]. Because the numerical model does not include water evaporation, it cannot capture the $\sim 100$ s delay in the thermocouples away from the igniter. The other phenomenon is the on-set of secondary charoxidation in the region near the inlet $(\mathrm{x}<60 \mathrm{~mm})$ at $400 \mathrm{~s}[18,6]$, which produces higher temperatures and higher $\mathrm{O}_{2}$ consumption. This reaction is not included in the 5-step mechanism and thus the model underpredicts the temperatures at those locations for $t>400 \mathrm{~s}$.

Results of the spatial profiles for the reaction rates, temperature and oxygen concentration at the smoldering front are presented in Fig. 4 (left for opposed, right for forward). It is seen that the model predicts that both fronts consume all the incoming oxygen. Considerable differences can be observed in the smolder-front structure for the two propagation modes. In opposed smoldering combustion (left of Fig. 4), the oxidation and the pyrolysis reactions overlap to form one single front. This is consistent with experimental observations, where the opposed-propagation front appears as one single smolder-front $[8$, 15]. The pyrolysis front combines contributions from the endothermic degradation of the foam and the $\beta$ foam. The oxidation front also has contributions from both, but it is dominated by the exothermic degradation of the $\beta$-foam. The starvation of oxygen occurring before the char oxidation is complete results in little heat provided to the front by this reaction, which is also in agreement with experimental 
observations $[8,15]$. The model predicts that both the pyrolysis and the oxidation fronts propagate at the same velocity in opposed smoldering combustion of PU. The structure in forward smoldering combustion (right of Fig. 4) is quite different. The oxidation and the pyrolysis reactions form two distinct propagating fronts: the pyrolysis front followed by the oxidation front. This result is also in agreement with experimental observations of forward propagation $[11,18]$. The pyrolysis front combines both the endothermic degradation of the foam and the $\beta$-foam, but the former dominates. Forward smoldering combustion results in virtually no oxidation of the virgin foam, as all of it is converted to $\beta$-foam via pyrolysis, but has energetic $\beta$-foam oxidation. The hot char region receives the fresh supply of oxidizer so the char oxidation is vigorous, and all the char is converted to solid residue. The model predicts that the pyrolysis front propagate faster than the oxidation front in forward configuration (about $0.07 \mathrm{~mm} / \mathrm{s}$ faster for $5 \mathrm{~mm} / \mathrm{s}$ inlet velocity). This finding was reported by Torero and Fernandez-Pello [11] as an observation in their experiments, and it is due to the thermal wave traveling at a faster velocity than the oxidation wave. The resulting structure of the propagating wave with this characteristic is called 'reaction trailing' [3].

The effect of the inlet airflow on the propagation velocities is presented in Fig. 5. Forward smoldering propagation is about $30 \%$ faster than opposed for the same inlet air velocity. The model predicts a sudden extinction of opposed smoldering combustion due to over-blowing. The results shown in Fig. 5 only applied to the particular ignition protocol implemented, i.e. the same as in $[8,18]$ but with the inlet forced-flow to its nominal value $u_{0}$ since $t=0$. It is expected that these results will change if the ignition protocol changes, especially for low airflow velocities.

\section{Concluding Remarks}

The model presented here accounts for the most complete description of the chemical reactions and transport mechanisms in smoldering combustion to date. Using a 5-step mechanism, the model of smoldering ignition and propagation in a porous media describes well both opposed and forward 
propagation. Specifically, the model predicts the reaction-front thermal and species structure, the onset of smoldering ignition, the propagation rate and the temperature profiles. The present model results, despite the inaccuracies, reproduce the most important features of the process and represent a major improvement in the modeling of smoldering combustion.

The fact that it is possible to predict the experimental observations in both opposed and forward propagation is a significant step forward in the development of numerical models of smoldering combustion. This is particularly relevant in multidimensional simulations where clear distinction between forward and opposed modes is no longer applicable.

\section{Acknowledgements}

This work was supported by the National Aeronautics and Space Administration, under grant NAG32026. The authors thank Prof. Jose L. Torero, Chris Lautenberger, Olivier Putzeys and Dr. Elizabeth Kallman for their help.

\section{References}

1. T.J. Ohlemiller, Progress Energy Combust. Sci. 11 (1985) 277-310.

2. T.J. Ohlemiller, J. Bellan, F.E. Rogers, Combust. Flame 36 (1979) 197-215.

3. D.A. Schult, B.J. Matkowsky, V.A. Volpert, A.C. Fernandez-Pello, Combust. Flame 104 (1996) 1-26.

4. J. Buckmaster, D. Lozinski, Combust. Flame 104 (1996) 300-310.

5. S.V. Leach, G. Rein, J.L. Ellzey, O.A. Ezekoye, J.L. Torero, Combust. Flame 120 (3) (2000) 346358.

6. G. Rein, A. Bar-Ilan, A.C. Fernandez-Pello, J.L. Ellzey, J.L. Torero, D.L. Urban, Proc. Combust. Inst. 30 (2005) 2327-2334.

7. M.L. Kelley, D.A. Schult, Combust. Theory Model. 10 (2006) 10(1) 133-143.

8. A. Bar-Ilan, G. Rein, D.C. Walther, A.C. Fernandez-Pello, J.L. Torero, D.L. Urban, Combust. Sci. Tech. 176 (2004) 2027-2055. 
9. T.J. Ohlemiller, D.A. Lucca, Combust. Flame 54 (1983) 131-147.

10. S.S. Dosanjh, P.J. Pagni, A.C. Fernandez-Pello, Combust. Flame 68 (1987) 131-142.

11. J.L. Torero, A.C. Fernandez-Pello, 1996, Combust. Flame 106 (1996) 89-109.

12. D.A. Schult, B.J. Matkowsky, V.A. Volpert, A.C. Fernandez-Pello, Combust. Flame 101 (19954) 471-490.

13. D. Lozinski, J. Buckmaster, Combust. Flame 102 (1995) 87-100.

14. S.S. Dosanjh, P.J. Pagni, A.C. Fernandez-Pello, Combust. Flame 68 (1987) 131-142.

15. J.L. Torero, A.C. Fernandez-Pello, M. Kitano, 1993, Combust. Sci. Tech 91 (1993) 95-117.

16. C. Di Blasi, Combust. Sci. Tech. 106 (1995) 103-124.

17. G. Rein, C. Lautenberger, A.C. Fernandez-Pello, J.L. Torero, D.L. Urban. Combust. Flame (2006) in press.

18. A. Bar-Ilan, G. Rein, A.C. Fernandez-Pello, J.L. Torero, D.L. Urban, Experiment. Therm. Fluid Sci. 28 (2004) 743-751.

19. G. Rein, Computational Model of Forward and Opposed Smoldering Combustion with Improved Chemical Kinetics, PhD. Thesis, Univ. of California at Berkeley (2005). Available at http://repositories.cdlib.org/cpl/fs/ReinPhD05.

20. R. Siegel, J.R. Howell, Thermal Radiation Heat Transfer, $3^{\text {rd }}$ ed., Taylor and Francis, New York, USA, 1992, p. 837.

21. F.E. Rogers, T.J. Ohlemiller, J. Fire Flamm. 11 (1980) 32-44.

22. D.C. Walther, A.C. Fernandez-Pello, D.L. Urban, Combust. Flame 116 (1999) 398-414.

23. P.N. Brown, G.D. Byrne, A.C. Hindmarsh, SIAM J. Sci. Stat. Comp. 10 (1989) 1038-1051.

24. Y. Sui-Hang, City University of Hong-Kong, personal communications about results to be submitted for publication (2005).

25. O. Putzeys, A. Bar-Ilan, G. Rein, A.C. Fernandez-Pello, D.L. Urban, Proc. Combust. Inst. 31 (2006) (in press).

26. J-W. Wu, W.-F. Sung, H.-S. Chu, Int. J. Heat Mass Transfer 42 (1999) 2211-2217. 


\section{Tables}

Table 1. Pore and fiber diameters measured from SEM photograph [19].

\begin{tabular}{lll}
\hline Foam & Pore $\mathrm{d}_{\mathrm{p}}$ & Fiber $\mathrm{d}_{\mathrm{f}}$ \\
\hline Virgin & $500 \mu \mathrm{m}$ & $85 \mu \mathrm{m}$ \\
Charred & $650 \mu \mathrm{m}$ & $50 \mu \mathrm{m}$ \\
\hline
\end{tabular}


Table 2. Value of the most important parameters used in the model

\begin{tabular}{cccc}
\hline Parameter & Value & Units & Reference \\
\hline $\mathrm{D}$ & 0.12 & $\mathrm{~m}$ & {$[8,18]$} \\
$\mathrm{L}$ & 0.14 & $\mathrm{~m}$ & {$[8,18]$} \\
$\rho_{0}$ & 30 & $\mathrm{~kg} / \mathrm{m}^{3}$ & {$[8,18]$} \\
$\phi_{0}$ & 0.97 & - & {$[8,18]$} \\
$\mathrm{c}_{\mathrm{s}}$ & 1760 & $\mathrm{~J} / \mathrm{kg}$ & {$[8,18]$} \\
$\mathrm{K}_{\mathrm{f}}$ & $5.210^{-9}$ & $\mathrm{~m}^{2}$ & {$[25]$} \\
$\mathrm{K}_{\mathrm{c}}$ & $310^{-8}$ & $\mathrm{~m}^{2}$ & {$[25]$} \\
$\mathrm{k}_{\mathrm{s}}$ & $3.410^{-2}$ & $\mathrm{~W} / \mathrm{mK}$ & {$[26]$} \\
$\Delta \mathrm{h}_{\mathrm{p}}$ & 40 & $\mathrm{~J} / \mathrm{g}-\mathrm{f}$ & this work \\
$\Delta \mathrm{h}_{\mathrm{p} \beta}$ & 750 & $\mathrm{~J} / \mathrm{g}-\beta$ & this work \\
$\Delta \mathrm{h}_{\mathrm{o}}$ & -1600 & $\mathrm{~J} / \mathrm{g}-\mathrm{f}$ & this work \\
$\Delta \mathrm{h}_{\mathrm{o} \beta}$ & -1850 & $\mathrm{~J} / \mathrm{g}-\beta$ & this work \\
$\Delta \mathrm{h}_{\mathrm{c}}$ & -2500 & $\mathrm{~J} / \mathrm{g}-\mathrm{c}$ & this work \\
$\mathrm{v}_{\mathrm{O}_{2}, \mathrm{o}}$ & 0.1 & $\mathrm{~g}-\mathrm{O}_{2} / \mathrm{g}-\mathrm{f}$ & this work \\
$\mathrm{v}_{\mathrm{O}_{2}, \mathrm{o} \beta}$ & 0.4 & $\mathrm{~g}-\mathrm{O}_{2} / \mathrm{g}-\beta$ & this work \\
$\mathrm{v}_{\mathrm{O}_{2}, \mathrm{c}}$ & 1.5 & $\mathrm{~g}-\mathrm{O}_{2} / \mathrm{g}-\mathrm{c}$ & this work \\
\hline
\end{tabular}




\section{Figure Captions}

Figure 1. One-dimensional domain for opposed and forward smoldering combustion.

Figure 2. Temperature profiles of the solid vs. distance from igniter at different times; left) opposed smoldering with an inlet airflow of $3 \mathrm{~mm} / \mathrm{s}$; and right) forward smoldering with an inlet airflow of 5 $\mathrm{mm} / \mathrm{s}$. Comparison of numerical results (line) with experimental results (circle with dashed line) $[8,18]$.

Figure 3. Temperature of the solid vs. time at different locations for; left) opposed smoldering with an inlet airflow of $3 \mathrm{~mm} / \mathrm{s}$; right) forward smoldering with an inlet airflow of $5 \mathrm{~mm} / \mathrm{s}$. Comparison of numerical (circles with line) with experimental results (line) $[8,18]$.

Figure 4. Numerical results for the front structure during self-propagation for; left) opposed smoldering with an inlet airflow of $3 \mathrm{~mm} / \mathrm{s}$; and right) forward smoldering with an inlet airflow of $5 \mathrm{~mm} / \mathrm{s}$. Top figures show the heat-released rate of each reaction (positive for oxidation, negative for pyrolysis). Bottom figures show the temperature and oxygen profiles.

Figure 5. Self-sustained propagation velocity of the smoldering front opposed and forward configurations. Filled circle means halfway quenched. 


\section{Figures}

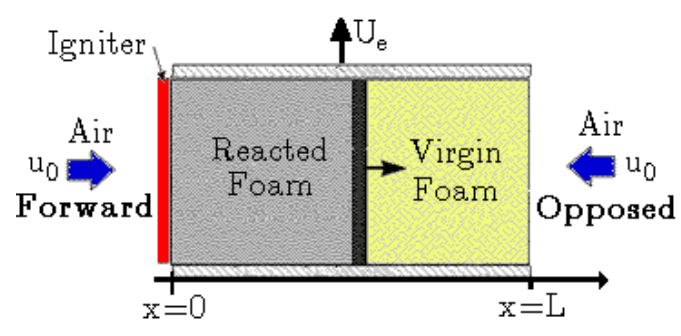

Figure 1 


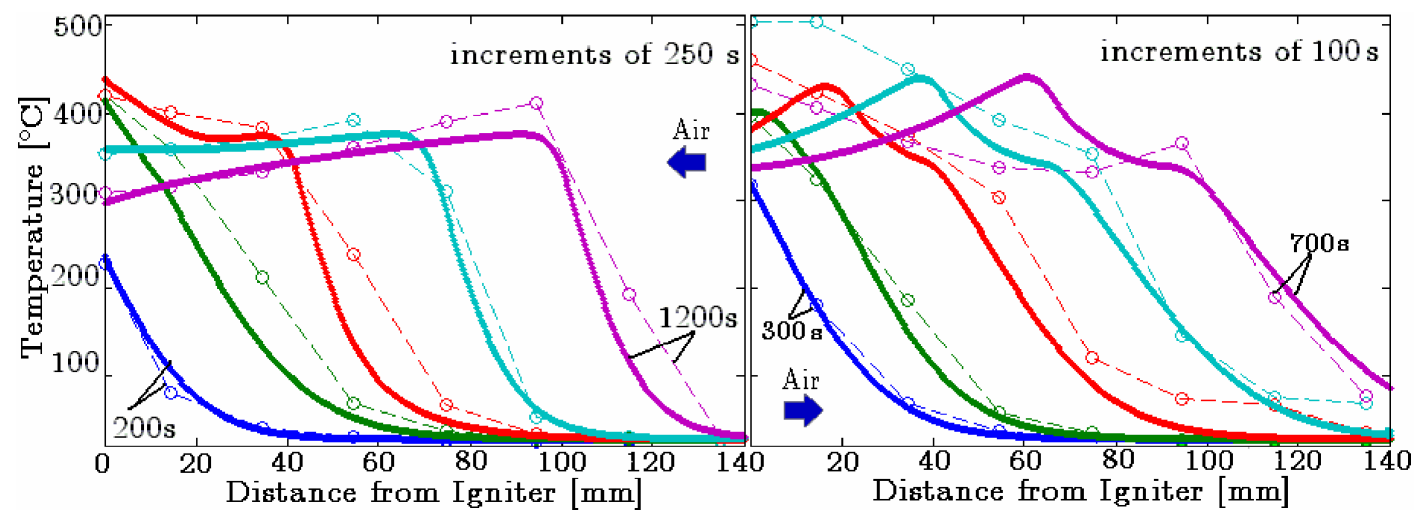

Figure 2 


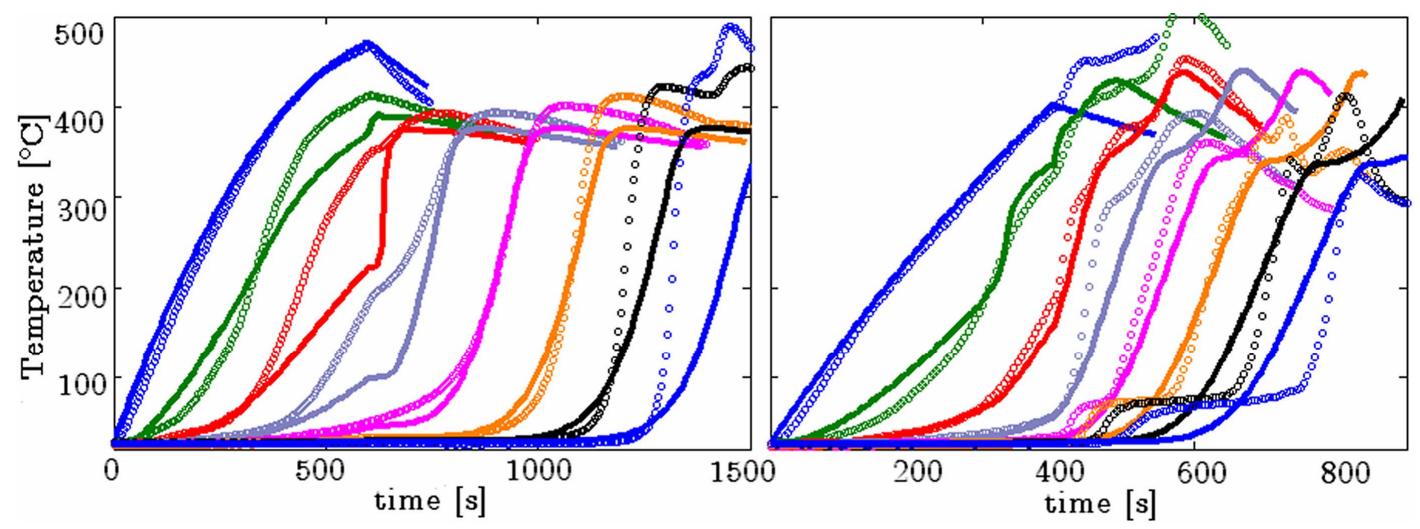

Figure 3 

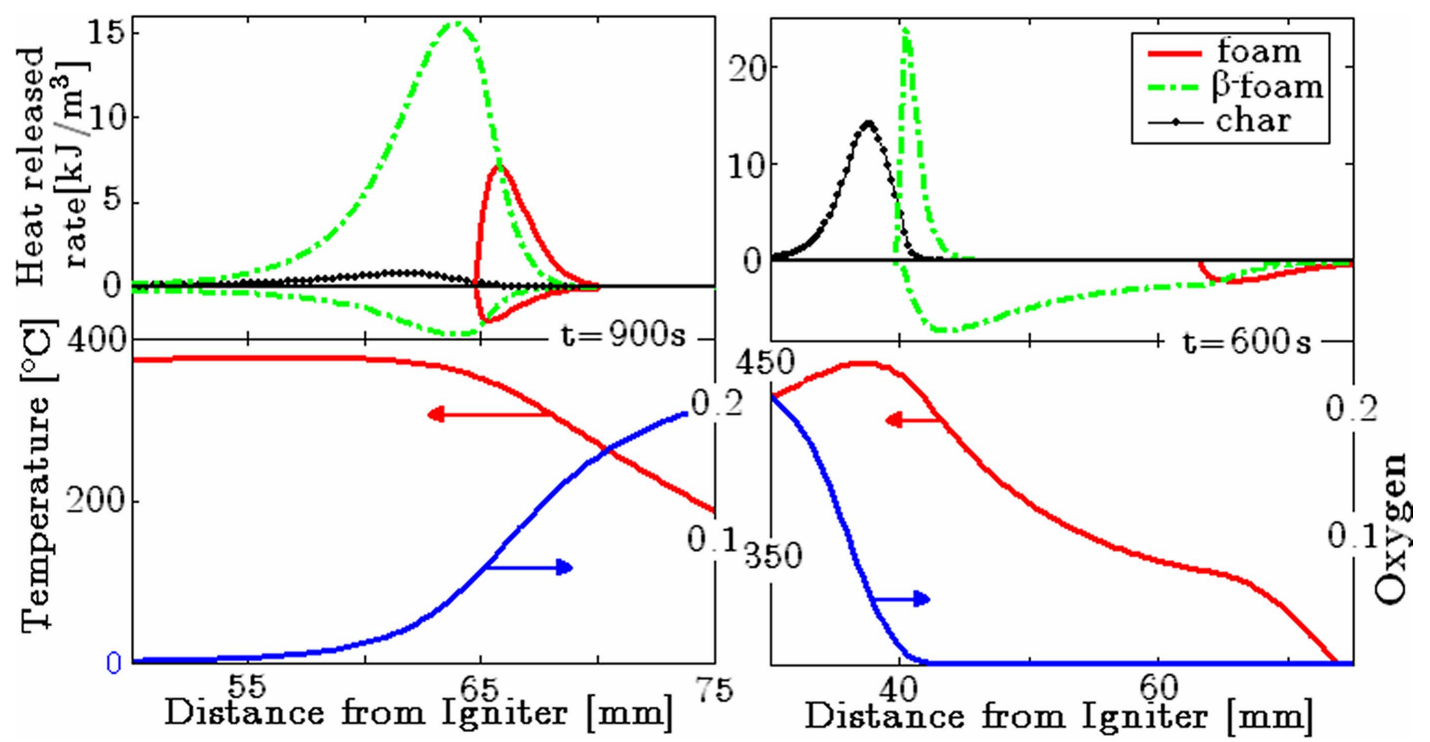

Figure 4 


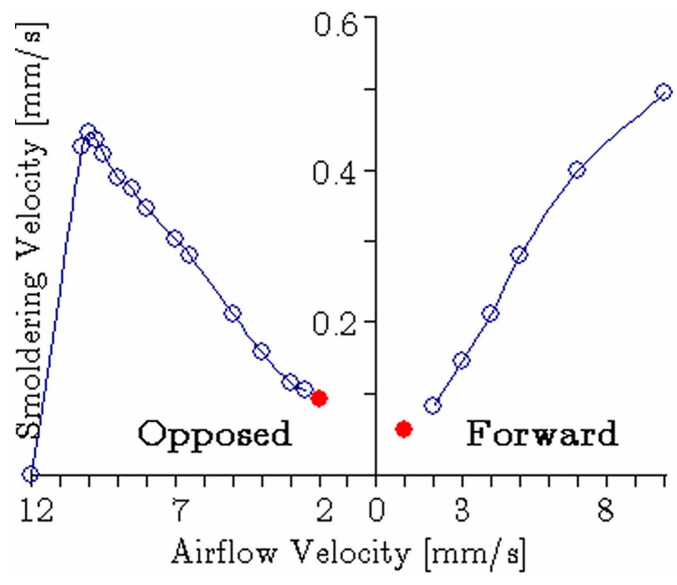

Figure 5. 